\title{
Les revues scientifiques en science de l'éducation: comment être singulier dans une offre plurielle?
}

\author{
Auteur(s) \\ Ugo Collard-Fortin, Université du Québec à Chicoutimi \\ ugo.collard-fortin@uqac.ca \\ Marie-Pierre Baron, Université du Québec à Chicoutimi, \\ marie-pierre baron@uqac.ca \\ Marie-Hélène Bruyère, Université du Québec à Montréal \\ bruyere.marie-helene@courrier.uqam.ca
}




\section{6}

\section{REVUE HYBRIDE DE L'ÉDUCATION}

\section{Mise en contexte}

Le monde de la communication est un domaine en constante évolution, notamment propulsé par les nombreuses avancées technologiques réalisées dans ce domaine. Bien que cet engouement offre des possibilités plus qu'intéressantes, il n'en demeure pas moins que la rapidité avec laquelle les changements surviennent au sein de celui-ci rend difficile la diffusion de l'information auprès de publics cibles, a fortiori celle ayant trait à la recherche. Les acteurs de l'édition scientifique et les organisations qu'ils représentent (e.g. revues scientifiques, organismes de liaison, etc.) doivent, dès lors, amorcer une réflexion sur les meilleurs moyens de diffusion à l'ère moderne. II importe d'emboiter le pas à cette réflexion en cours afin de faire émerger différents enjeux inhérents au processus du transfert des connaissances issues de la recherche et aux impératifs de la publication scientifique.

Nés avec l'avènement d'Internet et des technologies de l'information et de la communication, les auteurs de l'article sont témoins du changement de dynamique de la connaissance. Tel que rapporté par Fabre (2017), les connaissances sont aujourd'hui considérées comme un bien public et elles sont soumises à différents outils de partage et de mise en commun. Dans cet article de réflexion, voire même d'opinion, les auteurs se penchent donc sur différents enjeux du monde de l'éducation, soient ceux relatifs au transfert des connaissances issues de la recherche et ceux liés aux impératifs de la publication scientifique.

\section{Le transfert des connaissances issues de la recherche en éducation}

Longtemps, le domaine de la publication scientifique a été animé "par » et réfléchi "pour » les chercheurs universitaires. Toutefois, il importe de réfléchir la chose autrement, d'offrir une diffusion propice à une démocratisation des savoirs scientifiques s'adressant à un lectorat diversifié. L'utilisation des connaissances issues de la recherche (CIR) est un élément névralgique s'inscrivant dans cette préoccupation. Celle-ci est discutée au sein des domaines relevant des différentes sphères de la société (i.e. santé, services sociaux, justice, éducation). Dans le domaine de l'éducation, la plupart des acteurs concernés (e.g. enseignants, chercheurs, directeurs, décideurs politiques, etc.) s'entendent sur la grande potentialité d'une telle pratique, notamment pour influencer la réussite scolaire (Dagenais, Janosz, Abrami, et Bernard, 2009). Cela va de pair avec l'idée phare exposée dans le Mémoire du Conseil supérieur de l'éducation (CSE, 2016) dans le cadre des consultations publiques pour une politique de la réussite éducative qui défend, entre autres, un modèle de diffusion axé sur la mobilisation des CIR dans différents milieux.

[...] Les connaissances issues de la recherche doivent être partie intégrante des stratégies de développement professionnel continu déployées par le personnel enseignant. L'enjeu principal 


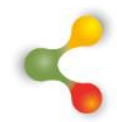

\section{REVUE HYBRIDE DE L'ÉDUCATION}

est de favoriser le transfert et la mobilisation des connaissances scientifiques par les acteurs de l'éducation pour le soutien à l'action en classe (accompagnement plus que contrôle). Cela permettrait de mieux cerner l'arrimage d'un projet d'organisme voué à la mise en valeur et au transfert des connaissances dans le milieu de l'éducation et de sa portée, entre autres, quant à l'espace d'autonomie des enseignants et le leadership pédagogique des directions d'écoles, des responsabilités professionnelles toutes deux inscrites dans la Loi sur l'instruction publique. Par ailleurs, la question de la responsabilité du transfert et de la mobilisation des connaissances pourrait être examinée, tant au regard de la tâche du personnel scolaire que de celle des chercheurs universitaires, pour lesquels le travail terrain auprès du personnel scolaire est peu reconnu (CSE, 2016, p.43).

Toutefois, le transfert des CIR en éducation est actuellement limité, car un décalage important subsiste entre les connaissances produites et celles qui sont réellement utilisées dans la pratique (Landry, Becheikh, Amara, Ziam, Idrissi et Castonguay, 2008; Williams et Cole, 2003), et ce, malgré une attitude foncièrement positive des acteurs concernés (e. g. administrateurs, enseignants, etc.) envers celles-ci (McNamara, 2002). À notre avis, les organisations de publication scientifique jouent un rôle important à l'intérieur de ce portrait, car, selon un récent rapport de recherche québécois portant sur l'utilisation des CIR chez les intervenants scolaires (Dagenais et al., 2010), certains facteurs liés au processus de communication et à la gestion de l'information sont à considérer. En réalité, l'avènement de l'ère numérique, et plus particulièrement d'Internet, a permis d'accroitre considérablement l'accès aux CIR, mais a également occasionné divers problèmes liés à la qualité, l'intelligibilité et l'abondance de l'information (Fabre, 2017 ; Landry et al., 2008). Cette dernière est donc accessible aux acteurs scolaires, mais demeure trop souvent dissimulée parmi des éléments superflus. Bach et Jérome (2014) reconnaissent le problème d'« isoler " l'information pertinente, en raison de la multiplication du nombre des articles dans des revues trop nombreuses et de qualité parfois médiocre. II se peut également que les textes et ouvrages produits par les chercheurs soient rédigés dans un vocable trop spécialisé et d'une rhétorique trop complexe pour les profanes. Les informations qu'ils contiennent demeurent alors inaccessibles aux praticiens ou encore apparaissent difficilement applicables en contexte scolaire. Bach et Jérome (2014) parlent d'une «crise de l'édition » pour décrire cette situation, qui remet en question rien de moins que le modèle même sur lequel est fondé l'ensemble du système d'information scientifique et technique au plan international.

\section{Les impératifs de la publication scientifique}

Le domaine de la publication scientifique est normé par un certain nombre de pratiques partagées par la plupart des organisations, dont 


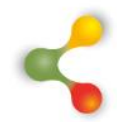

\section{REVUE HYBRIDE DE L'ÉDUCATION}

l'emploi de plateformes de diffusion (numériques ou non ; payantes ou en libre accès), l'attribution de facteurs d'impact et l'arbitrage ou l'évaluation par les pairs. Dans la situation actuelle, ces pratiques doivent être repensées afin d'être davantage en adéquation avec l'horizon scientifique contemporain.

Le libre accès est un enjeu de taille, car l'accessibilité est un catalyseur non négligeable de la chaine du transfert des CIR. Par exemple, il est raisonnable de penser qu'un praticien souhaitant consulter un article pouvant alimenter sa réflexion sur sa pratique se dissuadera de le faire s'il doit payer pour y avoir accès. Heureusement, l'accès immédiat, gratuit et universel aux résultats de la recherche scientifique est maintenant un objectif à privilégier, pour des raisons éthiques et pratiques fortes, partagées par la communauté scientifique et par les pouvoirs publics du monde entier (Bach et Jérome, 2014). Parallèlement, il faut aussi dire que le libre accès met en péril l'équilibre financier des revues scientifiques; il s'agit du point de croisement des enjeux scientifiques et économiques (Cartron, 2017). En réalité, l'édition scientifique est une entreprise dont la mise en marche, la coordination et la publicisation nécessitent beaucoup de temps et aussi, disons-le, d'argent (Baron, 2018). Dans le domaine de l'éducation, des partenaires privés soutiennent plus rarement ce genre d'organisations. Ce faisant, la recherche d'alternatives a stimulé l'emploi de plateformes numériques, permettant de faire des économies substantielles en plus d'assurer une certaine pérennité aux différentes revues. Le choix du libre accès induit donc, nous venons de l'évoquer, un passage de l'édition scientifique au numérique. D'ailleurs, la plupart des revues ont opté pour la voie numérique.

Une analyse des revues françaises en sciences humaines et sociales a d'ailleurs indiqué que « la moitié des revues sont diffusées en numérique sous abonnement avec barrière mobile et que $40 \%$ des revues ont choisi une diffusion numérique en accès ouvert » (Contat et Gremillet, 2015). Plus largement, en éducation, si quelques-unes des revues publiées en numérique par des éditeurs indépendants fonctionnent encore avec un abonnement restreint (entre 1 et 4 ans), beaucoup sont complètement en libre accès ou ont pris ce virage récemment. Toutefois, cette transition emporte dans son sillage plusieurs corolaires. En effet, dans un paysage où les médias sociaux occupent une place névralgique dans l'accès à l'information et où il faut se démarquer de ses concurrents, certaines revues mues par le souci de se rendre davantage visibles et crédibles ont choisi de mettre de l'avant l'utilisation de facteurs d'impact. Or, des observateurs tels que Guédon (2016) constatent une influence de celui-ci sur les processus de sélection et d'évaluation des articles soumis. Ainsi, le suivi de l'indice peut, par exemple, favoriser la sélection d'articles susceptibles d'attirer un maximum de lecteurs pour obtenir le maximum de citations et, par le fait même, entrainer des "mouvements de mode scientifique » (Guédon, 2016). Dès lors, la tendance est forte pour que les revues soient à la recherche d'articles qui auront le plus grand effet sur le 


\section{6}

\section{REVUE HYBRIDE DE L'ÉDUCATION}

calcul de cette métrique, et ce, au détriment de ceux moins en vogue, mais possiblement plus novateurs. Conjuguons à cela que l'arbitrage ou l'évaluation par les pairs est d'ores et déjà un processus difficile à franchir pour de nombreux auteurs ; la " qualité » d'une revue est d'ailleurs souvent corrélée à sa capacité de rejeter la grande majorité des articles soumis (ibid.). Un travail réellement novateur, différent, qui remet en question bon nombre de "vérités ", va heurter un certain nombre d'idées reçues. De ce fait, et tendanciellement, l'évaluation par les pairs peut exercer un rôle plutôt situé en faveur de la conservation du « paradigme » en place, pour paraphraser Thomas Kuhn. Alors, s'il est vrai que l'évaluation ou l'arbitrage par les pairs est nécessaire pour s'assurer de la valeur scientifique d'une contribution, il serait important du même souffle de préciser que celui-ci n'est pas l'apanage de cette valeur et que ce processus n'est pas non plus exempt de biais, d'influences externes, de limites, de paradoxes, etc. Celui qui, au final, paie le prix des ratés du processus d'arbitrage est, tout bien pesé, le lectorat qui ne peut avoir accès à des contributions qui demeurent d'intérêts social, éducatif et scientifique.

\section{Conclusions}

Alors que l'éducation est fondée sur le partage et la coconstruction de savoirs, de savoir-être et de savoir-faire, le monde de la publication privilégie principalement les publications dites scientifiques. Bien que l'accès universel et gratuit des CIR soit l'objectif à atteindre (Bach et Jérome, 2014), tout n'est pas gagné et il importe de faire des choix judicieux. Devant cette multitude de possibles dans le monde de la publication et devant les diverses contraintes exposées dans les enjeux, beaucoup est encore à réfléchir pour favoriser la diffusion des connaissances.

Dès lors, la communauté des sciences de l'éducation doit se questionner sur les meilleures manières de mettre en lumière les CIR à travers un flux d'informations en pleine croissance et de les rendre disponibles pour les praticiens, et ce, en favorisant une diffusion saine de l'information. II importe aussi, pour les éditeurs, d'envisager les possibilités offertes par différentes plateformes et différents formats de publication scientifique dans le but de favoriser et de maximiser l'utilisation des CIR par les praticiens, et ce, sans mettre en péril le fonctionnement des revues. Finalement, il importe de se demander de quelle manière une revue peut initier une discussion ouverte entre les chercheurs universitaires et les partenaires des milieux scolaires, créer une véritable synergie entre eux, et faire valoir, par cette interaction, les différents leviers pour engager une démarche de transformations des pratiques.

Aujourd'hui, beaucoup est à redéfinir et de nombreuses possibilités s'offrent aux acteurs de l'édition scientifique. Une réflexion collective sur les nouveaux enjeux de la publication s'impose pour que chacun puisse tirer son épingle du jeu, pour être singulier dans une offre plurielle. 


\section{REVUE HYBRIDE DE L'ÉDUCATION}

\section{Références}

Bach, J-F. et Jérôme, D. (2014). Les nouveaux enjeux de la production scientifique. Paris : Institut de France Académie des sciences.

Baron, M-P. (2018). La revue hybride de l'éducation: des chercheurs partenaires des milieux de pratique. Découvrir MagAcfas, Repéré à https://www.acfas.ca/publications/decouvrir/2018/06/revuehybride-education-chercheurs-partenaires-milieux-pratique

Cartron, E. (2017). Où se situent les enjeux actuels pour les productions scientifiques des sciences infirmières ?. Recherche en soins infirmiers 1(128), 5-5. doi : 10.3917/rsi.128.0005

Contat, O. et Gremillet, A.-S. (2015). Publier : à quel prix? Étude sur la structuration des coûts de publication pour les revues françaises en SHS. Revue française des sciences de l'information et de la communication, 7, 1-21. doi : 10.4000/rfsic. 1716

Dagenais, C., Janosz, M., Abrami, P. et Bernard, R., (2009). Examen des mécanismes en jeu dans la décision des intervenants scolaires d'utiliser les connaissances issues de la recherche pour changer les pratiques (Rapport de recherche intégral). Québec : CTREQ

Fabre, R. (2017). Les nouveaux enjeux de la connaissance: dynamiques numériques de l'accès et du partage. London : ISTE editions

Guedon, J-C. (2016). Du libre accès à la littérature scientifique et quelques enjeux de la recherche en contexte de développement. Dans F. Piron, S. Regulus et M.S. Dibounje Madiba (dir.), Justice cognitive, libre accès et savoirs locaux. Pour une science ouverte juste, au service du développement local durable. Québec, Éditions science et bien commun. Repéré à https://scienceetbiencommun.pressbooks.pub/justicecognitive1/ch apter/du-libre-acces-a-la-litterature-scientifique-et-de-quelquesenjeux-de-la-recherche-en-contexte-de-developpement/

Landry, R., Becheikh, N., Amara, N., Ziam, S., Idrissi, O. et Castonguay, Y. (2008). La recherche, comment s'y retrouver? Revue systématique des écrits sur le transfert de connaissances en éducation. Chaire FCRSS/IRSC sur le transfert de connaissances et l'innovation, Québec : Université Laval.

McNamara, O. (2002). Becoming an evidance-based practitioner: A framework to teacher-researchers. London; New York: Routledge/Falmer. 


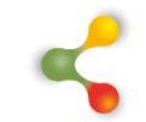

\section{REVUE HYBRIDE DE L'ÉDUCATION}

Williams, D. et Cole, L. (2003). The use of research by teachers : information literacy access and attitudes. Final report on the study funded by ESRC. ESRC 